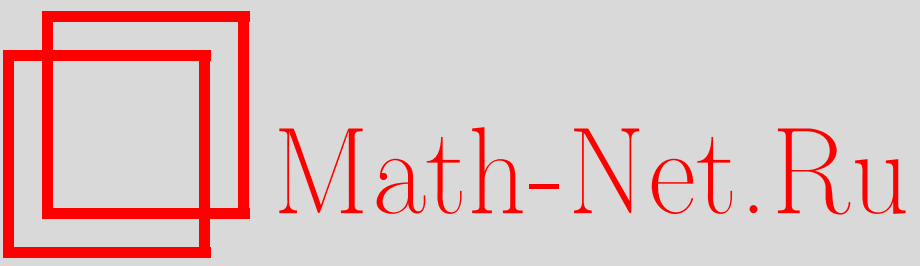

Х. Какухата, К. Конно, Вращающийся петлевой солитон в связанных бездисперсионных уравнениях, ТМФ, 2002, том 133, номер 3, 419-428

DOI: https://doi.org/10.4213/tmf408

Использование Общероссийского математического портала Math-Net.Ru подразумевает, что вы прочитали и согласны с пользовательским соглашением

http://www.mathnet.ru/rus/agreement

Параметры загрузки:

IP: 35.173 .219 .12

26 апреля 2023 г., 16:16:30 
ТЕОРЕТИЧЕСКАЯ

И МАТЕМАТИЧЕСКАЯ

ФИЗИКА

Том 133, № 3

декабрь, 2002

(C) 2002 г.

Х. Какухата*, К. Конно

\section{ВРАЩАЮШИЙСЯ ПЕТЛЕВОЙ СОЛИТОН В СВЯЗАННЫХ БЕЗДИСПЕРСИОННЫХ УРАВНЕНИЯХ}

Исследуются солитонные решения связанных бездисперсионных уравнений, которые описывают токоподводяшую струну, взаимодействуюшую с внешним магнитным полем в трехмерном евклидовом пространстве, посредством билинейных уравнений. Получен новый тип петлевых солитонных решений, которые врашаются вокруг $Z$-оси. Исследуется также двухсолитонное взаимодействие.

Ключевые слова: модель струны, бездисперсионные уравнения, петлевые солитоны, вращающийся солитон.

\section{1. ВВЕДЕНИЕ}

Связанные бездисперсионные уравнения имеют глубокие связи с алгебрами Ли [1] и обладают гамильтоновыми структурами [2]. Если задана одна алгебра Ли, то имеется система бездисперсионных уравнений, обладаюших гамильтоновой структурой и допускаюших применение метода обратной задачи рассеяния. Одним из простейших является случай $s u(2)$ :

$$
\begin{aligned}
q_{x t}+\frac{1}{2}\left(r^{*} r\right)_{x} & =0, \\
r_{x t}-q_{x} r & =0, \\
r_{x t}^{*}-q_{x} r^{*} & =0,
\end{aligned}
$$

где нижний индекс обозначает производные, а звездочка - комплексное сопряжение. $N$-солитонное решение такой системы получается с помошью метода обратной задачи рассеяния [3]. Недавно мы предложили физическое применение указанных уравнений, описывающих токоподводяшую струну в некотором внешнем магнитном поле [4]. Основываясь на этой точке зрения, с помощью билинейного преобразования мы получили одно- и двухсолитонные решения. Солитоны демонстрируют петлевые формы в трехмерном евклидовом пространстве $\mathbb{R}^{3}$. Закон сохранения момента импульса может быть

\footnotetext{
* Toyama University, Toyama, Japan

${ }^{\dagger}$ Department of Physics, College of Science and Technology, Nihon University, Tokyo, Japan
} 
выведен из уравнений движения струны, так что можно ожидать появления вращающихся петлевых солитонов. Однако приведенные в работе [4] солитоны имеют нулевые моменты импульса. В настоящей работе мы приводим новый тип петлевых солитонных решений связанных бездисперсионных уравнений, имеюших ненулевой момент импульса, и рассматриваем взаимодействия между такими солитонами. В качестве первого шага будет дан краткий обзор связанных бездисперсионных уравнений, где солитоны распространяются в плоскости. После этого мы выведем петлевые одно- и двухсолитонные решения вращающегося типа в трехмерии с помощью модификации билинейного преобразования. Затем обсуждаются двухсолитонные взаимодействия. Последний раздел посвящен заключительным замечаниям.

\section{2. СВЯЗАННЫЕ БЕЗДИСПЕРСИОННЫЕ УРАВНЕНИЯ И ПЕТЛЕВЫЕ СОЛИТОНЫ}

Уравнения (1) принимают вид уравнений типа нелинейных уравнений Клейна-Гордона

$$
\begin{aligned}
X_{\tau \tau}-X_{\sigma \sigma} & =-\left(Z_{\tau}+Z_{\sigma}\right) X \\
Y_{\tau \tau}-Y_{\sigma \sigma} & =-\left(Z_{\tau}+Z_{\sigma}\right) Y, \\
Z_{\tau \tau}-Z_{\sigma \sigma} & =\left(X_{\tau}+X_{\sigma}\right) X+\left(Y_{\tau}+Y_{\sigma}\right) Y,
\end{aligned}
$$

если выполнить преобразования зависимых и независимых переменных

$$
\begin{array}{ll}
q=-Z, & r=X+i Y \\
\tau=x+t, & \sigma=x-t .
\end{array}
$$

Вводя векторы $\mathbf{r}=(X, Y, Z)$ и $\mathbf{J}=(0,0,1)$, можно переписать уравнения (2) в виде

$$
\mathbf{r}_{\tau \tau}-\mathbf{r}_{\sigma \sigma}=\left(\mathbf{r}_{\tau}+\mathbf{r}_{\sigma}\right) \times(\mathbf{J} \times \mathbf{r})
$$

Поскольку $\mathbf{B}$, определяемое как $\mathbf{J} \times \mathbf{r}$, удовлетворяет уравнениям Максвелла

$$
\begin{aligned}
\operatorname{rot} \mathbf{B} & =2 \mathbf{J}, \\
\operatorname{div} \mathbf{B} & =0,
\end{aligned}
$$

получаем, что В порождается постоянным электрическим током Ј. Здесь мы считаем, что $\tau$ - время, $\sigma$ - длина дуги струны и $\mathbf{r}$ - вектор положения струны.

Множитель $\mathbf{r}_{\tau}+\mathbf{r}_{\sigma}$ в правой части уравнения (5) можно интерпретировать как силу Лоренца, действуюшую на эффективный внутренний ток, где $\mathbf{r}_{\sigma}-$ внутренний электрический ток, а $\mathbf{r}_{\tau}$ - поправочный член, индуцированный движением струны к $\mathbf{r}_{\sigma}$. Уравнение (5) может поэтому представлять токоподводяшую струну, взаимодействующую с внешним магнитным полем $\mathbf{J} \times \mathbf{r}$. Статический случай уравнения (5) показывает, что в отличие от уравнений типа Кд $\Phi$, в которых дисперсионный эффект уравновешивает нелинейность, нелинейная внешняя сила в бездисперсионных уравнениях уравновешивает линейную упругость струны. 
Для того чтобы получить солитоные решения при граничном условии $\mathbf{r} \rightarrow(0,0, \sigma)$, когда $|\sigma| \rightarrow \infty$, мы билинеаризуем уравнения (2):

$$
\begin{aligned}
\left(D_{\tau}^{2}-D_{\sigma}^{2}+1\right) F \cdot G & =0, \\
\left(D_{\tau}^{2}-D_{\sigma}^{2}+1\right) F \cdot H & =0, \\
\left(D_{\tau}-D_{\sigma}\right)^{2} F \cdot F-\frac{1}{2}\left(G^{2}+H^{2}\right) & =0
\end{aligned}
$$

с помошью преобразования

$$
X=\frac{G}{F}, \quad Y=\frac{H}{F}, \quad Z=\sigma+2\left(\partial_{\tau}-\partial_{\sigma}\right) \ln F,
$$

где $D$ - производная Хироты. Впервые этот тип билинейных уравнений был выведен Алагесаном и Порсезяном [5]. К сожалению, им не удалось получить известные солитонные решения из-за отсутствия первого слагаемого $\sigma$ в последнем из уравнений (8).

Согласно обычной пертурбативной процедуре, можно получить односолитонное решение

$$
\begin{aligned}
& F=1+B e^{2 \eta}, \\
& G=\cos \Theta \cdot e^{\eta}, \\
& H=\sin \Theta \cdot e^{\eta},
\end{aligned}
$$

где $\Theta$ - угловой параметр, фаза равна $\eta=\omega \tau+p \sigma+\delta$, дисперсионное соотношение имеет вид

$$
\omega^{2}-p^{2}=-1,
$$

а коэффициент должен быть равным $B=1 /\left[16(\omega-p)^{2}\right]$.

$\mathrm{B}$ физическом пространстве $\mathbb{R}^{3}$ односолитонное решение имеет вид

$$
\begin{aligned}
& X=2 \sqrt{\frac{1+v}{1-v}} \cos \Theta \operatorname{sech} \frac{\sigma-v \tau}{\sqrt{1-v^{2}}} \\
& Y=2 \sqrt{\frac{1+v}{1-v}} \sin \Theta \operatorname{sech} \frac{\sigma-v \tau}{\sqrt{1-v^{2}}}, \\
& Z=Z_{0}+\sigma-2 \sqrt{\frac{1+v}{1-v}} \operatorname{th} \frac{\sigma-v \tau}{\sqrt{1-v^{2}}}
\end{aligned}
$$

при преобразовании

$$
\begin{gathered}
\omega=-\frac{v}{\sqrt{1-v^{2}}}, \\
p=\frac{1}{\sqrt{1-v^{2}}},
\end{gathered}
$$

где $v$ - фазовая скорость солитона такая, что $-1<v<1$, а $Z_{0}$ - константа. Это решение представляет собой солитон в форме петли, движущейся в плоскости, расположенной под углом $\Theta$ к плоскости $X Z$. Ясно, что амплитуда солитона возрастает при возрастании $v$. 
Двухсолитонное решение имеет вид

$$
\begin{aligned}
& F=1+B_{1} e^{2 \eta_{1}}+B_{2} e^{2 \eta_{2}}+B_{12} e^{\eta_{1}+\eta_{2}}+E_{12} e^{2\left(\eta_{1}+\eta_{2}\right)}, \\
& G=\cos \Theta_{1} \cdot e^{\eta_{1}}+\cos \Theta_{2} \cdot e^{\eta_{2}}+C_{G 1} e^{2 \eta_{1}+\eta_{2}}+C_{G 2} e^{\eta_{1}+2 \eta_{2}}, \\
& H=\sin \Theta_{1} \cdot e^{\eta_{1}}+\sin \Theta_{2} \cdot e^{\eta_{2}}+C_{H 1} e^{2 \eta_{1}+\eta_{2}}+C_{H 2} e^{\eta_{1}+2 \eta_{2}},
\end{aligned}
$$

где коэффициенты даются выражениями

$$
\begin{aligned}
B_{n} & =\frac{1}{16\left(\omega_{n}-p_{n}\right)^{2}}, \quad n=1,2, \\
B_{12} & =\frac{\cos \left(\Theta_{1}-\Theta_{2}\right)}{2\left(\omega_{1}+\omega_{2}-p_{1}-p_{2}\right)^{2}}, \\
C_{G 1} & =-\cos \Theta_{2} \frac{\left(\omega_{1}-\omega_{2}\right)^{2}-\left(p_{1}-p_{2}\right)^{2}}{\left(\omega_{1}+\omega_{2}\right)^{2}-\left(p_{1}+p_{2}\right)^{2}} B_{1}, \\
C_{G 2} & =-\cos \Theta_{1} \frac{\left(\omega_{1}-\omega_{2}\right)^{2}-\left(p_{1}-p_{2}\right)^{2}}{\left(\omega_{1}+\omega_{2}\right)^{2}-\left(p_{1}+p_{2}\right)^{2}} B_{2}, \\
C_{H 1} & =-\sin \Theta_{2} \frac{\left(\omega_{1}-\omega_{2}\right)^{2}-\left(p_{1}-p_{2}\right)^{2}}{\left(\omega_{1}+\omega_{2}\right)^{2}-\left(p_{1}+p_{2}\right)^{2}} B_{1}, \\
C_{H 2} & =-\sin \Theta_{1} \frac{\left(\omega_{1}-\omega_{2}\right)^{2}-\left(p_{1}-p_{2}\right)^{2}}{\left(\omega_{1}+\omega_{2}\right)^{2}-\left(p_{1}+p_{2}\right)^{2}} B_{2}, \\
E_{12} & =\left[\frac{\left(\omega_{1}-\omega_{2}\right)^{2}-\left(p_{1}-p_{2}\right)^{2}}{\left(\omega_{1}+\omega_{2}\right)^{2}-\left(p_{1}+p_{2}\right)^{2}}\right]^{2} B_{1} B_{2},
\end{aligned}
$$

а фазы равны $\eta_{n}=\omega_{n} \tau+p_{n} \sigma+\delta_{n}, n=1,2$.

Исходя из решения (13), взаимодействия неврашающихся солитонов можно разделить на три типа, которые обычно обнаруживаются в случае нахождения обоих солитонов в одной плоскости:

- петли не пересекаются и толкают друг друга с небольшой относительной скоростью (см. рис. $1 \mathrm{a} ; v=0.12$ ),

- небольшая петля движется вокруг большой с сушественной относительной скоростью (см. рис. $1 б ; ~ v=0.24$ ),

- небольшая петля исчезает в течение периода взаимодействия, а большая увеличивает свою амплитуду при столкновении положительной и отрицательной амплитуд (см. рис. $1 \mathrm{~B} ; v=0.24)$.

Каждая фазовая скорость выбирается в том же виде, что и в уравнениях (12). Типы взаимодействия аналогичны описанным в работе [6], за исключением последнего. В первых двух типах формы петель сохраняются в ходе взаимодействия, однако в последнем случае этого не происходит.

\section{3. ВРАШАЮШИЙСЯ ПЕТЛЕВОЙ СОЛИТОН}

Используя уравнения (2), легко показать, что $Z$-компонента момента импульса $\int\left(\mathbf{r}_{\tau} \times\right.$ $\mathbf{r}) d \sigma$ является сохраняюшейся величиной. Хотя петлевой солитон может иметь ненулевой момент импульса, решение (13) имеет нулевой момент импульса. В этом разделе мы 


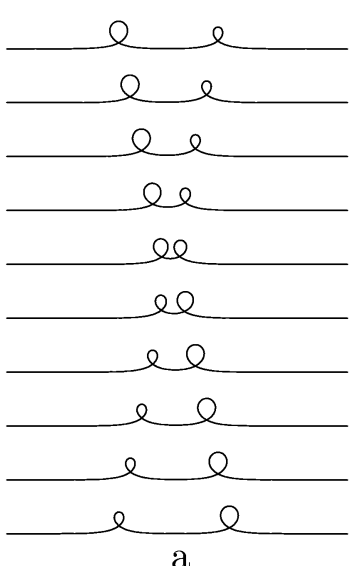

a
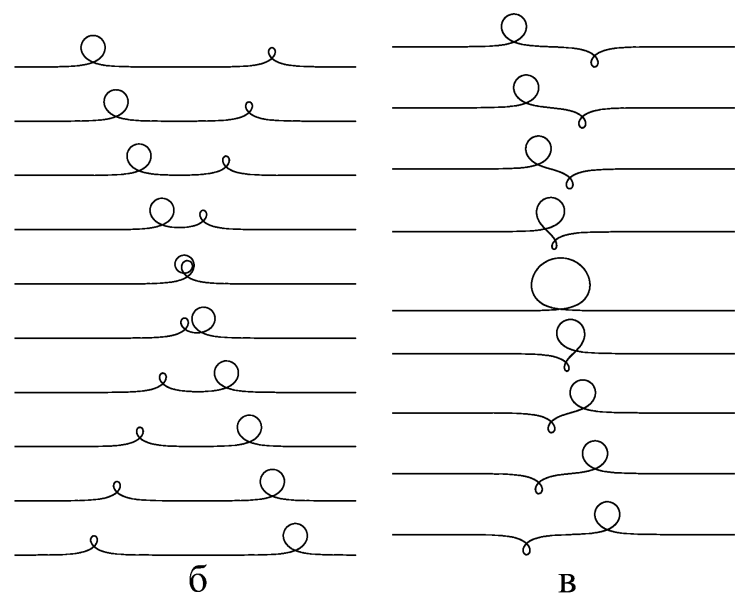

Рис. 1. Лобовое столкновение двух петлевых солитонов, движущихся в двумерном пространстве с одинаковыми скоростями $v$, но в противоположных направлениях.

выведем решения с ненулевыми моментами импульса, т.е. вращаюшиеся солитоны. Для этого удобно ввести комплексную переменную $Q=G+i H$. Тогда билинейные уравнения (7) можно переписать в виде

$$
\begin{aligned}
\left(D_{\tau}^{2}-D_{\sigma}^{2}+1\right) F \cdot Q & =0, \\
\left(D_{\tau}-D_{\sigma}\right)^{2} F \cdot F-\frac{1}{2} Q^{*} Q & =0 .
\end{aligned}
$$

Следуя обычной процедуре, разложим $F$ и $Q$ в формальный степенной ряд по вещественному параметру $\varepsilon$ :

$$
\begin{aligned}
& F=1+\varepsilon^{2} f_{2}+\varepsilon^{4} f_{4}+\varepsilon^{6} f_{6}+\cdots, \\
& Q=\varepsilon g_{1}+\varepsilon^{3} g_{3}+\varepsilon^{5} g_{5}+\cdots .
\end{aligned}
$$

Можно получить односолитонное решение уравнения (15) в виде

$$
\begin{aligned}
& F=1+B e^{\eta^{*}+\eta} \\
& Q=e^{\eta}
\end{aligned}
$$

где фаза равна

$$
\eta=k \sigma+\omega \tau+\delta+i \theta
$$

$k$ - комплексное волновое число, $\omega$ - комплексная частота, $\delta$ и $\theta$ - вешественные начальные фазы. Дисперсионное соотношение в этом случае то же, что в уравнении (10). Используя вешественные параметры, разложим фазу на вешественную $\left(\eta_{R}\right)$ и мнимую $\left(\eta_{I}\right)$ части:

$$
\eta=\eta_{R}+i \eta_{I}
$$



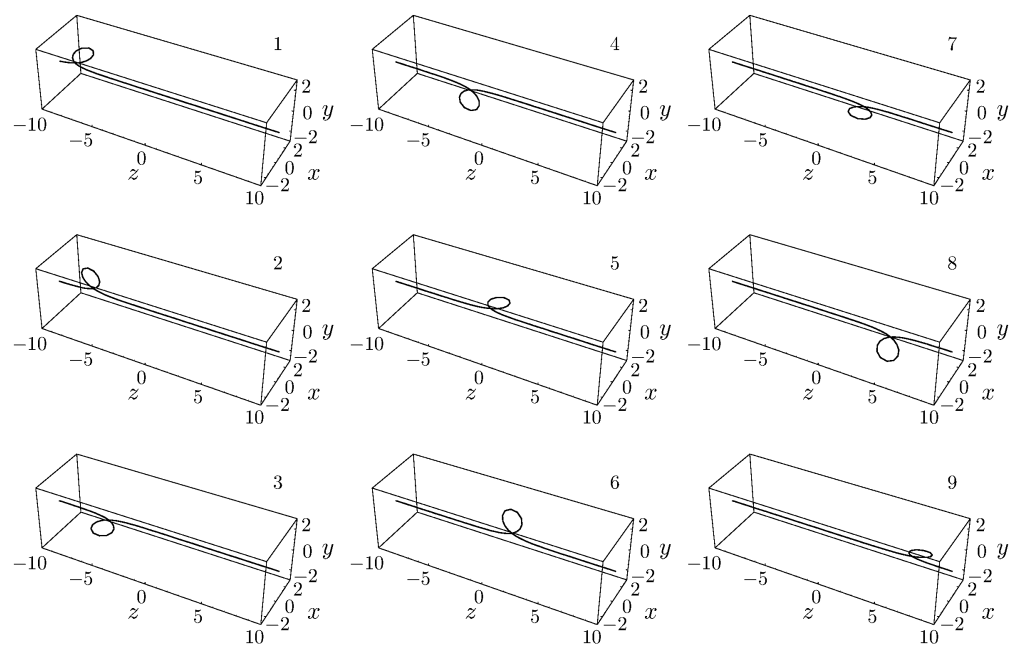

Рис. 2. Односолитонное решение при $v=0.24$ и $\Omega=0.2$.

где

$$
\begin{aligned}
\eta_{R} & =k_{R} \sigma+\omega_{R} \tau+\delta, \\
\eta_{I} & =k_{I} \sigma+\omega_{I} \tau+\theta .
\end{aligned}
$$

При таких параметрах дисперсионное соотношение принимает вид

$$
\begin{aligned}
\omega_{R}^{2}-k_{R}^{2}-\omega_{I}^{2}+k_{I}^{2} & =-1, \\
\omega_{R} \omega_{I}-k_{R} k_{I} & =0 .
\end{aligned}
$$

$\mathrm{B} \mathbb{R}^{3}$ после преобразований

$$
\begin{aligned}
\omega_{R} & =-\sqrt{\frac{1-\left(1-v^{2}\right) \Omega^{2}}{1-v^{2}}} v, & k_{R} & =\sqrt{\frac{1-\left(1-v^{2}\right) \Omega^{2}}{1-v^{2}},} \\
\omega_{I} & =\Omega, & k_{I} & =-v \Omega
\end{aligned}
$$

односолитонное решение имеет вид

$$
\begin{aligned}
& X=A \cos \eta_{I} \operatorname{sech} \eta_{R}, \\
& Y=A \sin \eta_{I} \operatorname{sech} \eta_{R}, \\
& Z=Z_{0}+\sigma-A \operatorname{th} \eta_{R},
\end{aligned}
$$

где $Z_{0}$ - константа, амплитуда $A$ имеет вид

$$
A=2 \sqrt{1-\left(1-v^{2}\right) \Omega^{2}} \sqrt{\frac{1+v}{1-v}}
$$

и

$$
\begin{aligned}
\eta_{R} & =\sqrt{\frac{1-\left(1-v^{2}\right) \Omega^{2}}{1-v^{2}}}(\sigma-v \tau)+\delta, \\
\eta_{I} & =\Omega(\tau-v \sigma)+\theta .
\end{aligned}
$$


Здесь $v$ и $\Omega$ соответственно фазовая и угловая скорости солитона, удовлетворяющие условиям

$$
\begin{gathered}
-1<v<1 \\
\frac{-1}{\sqrt{1-v^{2}}}<\Omega<\frac{1}{\sqrt{1-v^{2}}} .
\end{gathered}
$$

Это решение описывает петлевые солитоны, которые вращаются вокруг оси $Z$ (рис. 2).

Поскольку эффект врашения солитона выражается в появлении дополнительного множителя $\sqrt{1-\left(1-v^{2}\right) \Omega^{2}}$ в выражениях для амплитуды $A$ и фазы $\eta_{R}$, петлевая амплитуда меньше соответствуюшей амплитуды неврашаюшегося солитона.

Двухсолитонное решение имеет вид

$$
\begin{aligned}
& F=1+b_{1} e^{\eta_{1}^{*}+\eta_{1}}+b_{12}^{*} e^{\eta_{1}^{*}+\eta_{2}}+b_{12} e^{\eta_{1}+\eta_{2}^{*}}+b_{2} e^{\eta_{2}^{*}+\eta_{2}}+d_{12} e^{\eta_{1}^{*}+\eta_{1}+\eta_{2}^{*}+\eta_{2}} \\
& Q=e^{\eta_{1}}+e^{\eta_{2}}+c_{1} e^{\eta_{1}^{*}+\eta_{1}+\eta_{2}}+c_{2} e^{\eta_{1}+\eta_{2}^{*}+\eta_{2}}
\end{aligned}
$$

где коэффициенты равны

$$
\begin{aligned}
b_{n} & =\frac{1}{4\left(\omega_{n}^{*}+\omega_{n}-k_{n}^{*}-k_{n}\right)^{2}}, \quad n=1,2 \\
b_{12} & =\frac{1}{4\left(\omega_{1}+\omega_{2}^{*}-k_{1}-k_{2}^{*}\right)^{2}}, \\
c_{1} & =4\left(\omega_{1}-\omega_{2}-k_{1}+k_{2}\right)^{2} b_{1} b_{12}^{*} \\
c_{2} & =4\left(\omega_{1}-\omega_{2}-k_{1}+k_{2}\right)^{2} b_{2} b_{12} \\
d_{12} & =16\left|\omega_{1}-\omega_{2}-k_{1}+k_{2}\right|^{4} b_{1} b_{2}\left|b_{12}\right|^{2}
\end{aligned}
$$

а фазы суть $\eta_{n}=k_{n} \sigma+\omega_{n} \tau+\delta_{n}+i \theta_{n}$, причем дисперсионное соотношение имеет вид

$$
\omega_{n}^{2}-k_{n}^{2}=-1
$$

при $n=1,2$. Сдвиги фаз задаются выражениями

$$
\begin{aligned}
& \operatorname{Re}\left(\phi_{1}\right)=-\frac{1}{2} \ln \frac{d_{12}}{b_{1} b_{2}}+\left(k_{1}^{*}+k_{1}\right)\left(\omega_{2}^{*}+\omega_{2}-k_{2}^{*}-k_{2}\right), \\
& \operatorname{Im}\left(\phi_{1}\right)=i \ln \frac{\left(\omega_{1}-\omega_{2}-k_{1}+k_{2}\right)^{2} b_{12}}{\left|\omega_{1}-\omega_{2}-k_{1}+k_{2}\right|^{2}\left|b_{12}\right|}
\end{aligned}
$$

для первого солитона и

$$
\begin{aligned}
& \operatorname{Re}\left(\phi_{2}\right)=\frac{1}{2} \ln \frac{d_{12}}{b_{1} b_{2}}+\left(k_{2}^{*}+k_{2}\right)\left(\omega_{1}^{*}+\omega_{1}-k_{1}^{*}-k_{1}\right), \\
& \operatorname{Im}\left(\phi_{2}\right)=-i \ln \frac{\left(\omega_{1}-\omega_{2}-k_{1}+k_{2}\right)^{2} b_{12}^{*}}{\left|\omega_{1}-\omega_{2}-k_{1}+k_{2}\right|^{2}\left|b_{12}\right|}
\end{aligned}
$$

для второго солитона. Первые члены в правых частях первых из уравнений (30) и (31) показывают фазовые сдвиги, задаваемые нелинейным взаимодействием между солитонами, а вторые члены представляют собой фазовые сдвиги, причиной которых являются 
расталкивание двумя петлями друг друга или движение вдоль другой петли. Вторые уравнения представляют собой врашательные фазовые сдвиги.

Рассмотрим двухсолитонное взаимодействие. Согласно уравнениям (20) и (25)

$$
\begin{gathered}
\eta_{n R}=k_{n R} \sigma+\omega_{n R} \tau+\delta_{n}, \\
\eta_{n I}=k_{n I} \sigma+\omega_{n I} \tau+\theta_{n}, \\
\omega_{n R}=-\sqrt{\frac{1-\left(1-v_{n}^{2}\right) \Omega_{n}^{2}}{1-v_{n}^{2}}} v_{n}, \quad k_{n R}=\sqrt{\frac{1-\left(1-v_{n}^{2}\right) \Omega_{n}^{2}}{1-v_{n}^{2}}}, \\
\omega_{n I}=\Omega_{n}, \\
k_{n I}=-v_{n} \Omega_{n},
\end{gathered}
$$

где $n=1,2$. Здесь мы рассмотрим специальные случаи лобового столкновения с одинаковыми, но противоположно направленными скоростями $v$ и взаимодействие вращающегося петлевого солитона с покоящимся. Как показано на рис. 3, лобовое взаимодействие происходит между солитонами, вращаюшимися вокруг оси $Z$ трехмерным образом. При небольшой относительной скорости солитоны обмениваются своими амплитудами и угловыми скоростями, так что они расталкивают друг друга (см. рис. $3 \mathrm{a} ; v=v_{1}=$ $\left.-v_{2}=0.12, \Omega_{1}=-0.1, \Omega_{2}=0.1\right)$. При большой относительной скорости малый солитон движется вдоль большой петли (см. рис. $3 б ; v=0.36, \Omega_{1}=-0.1, \Omega_{2}=0.1$ ). Искажения солитона больше, чем при взаимодействии неврашаюшихся солитонов.
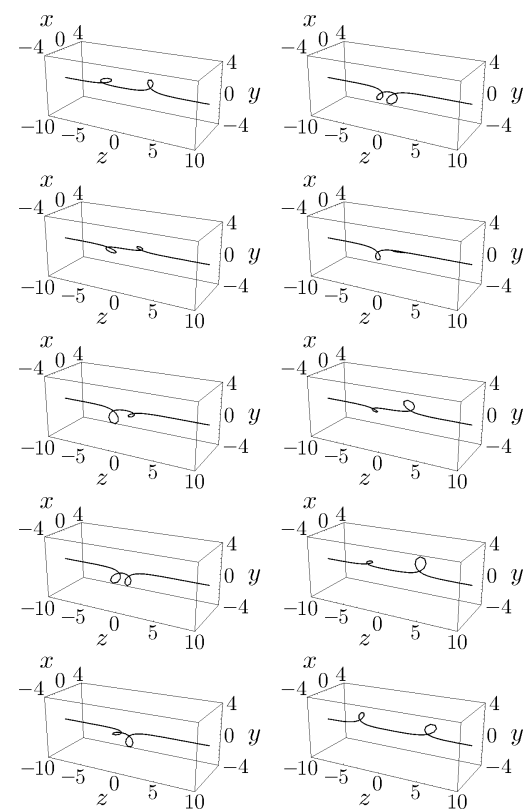

a
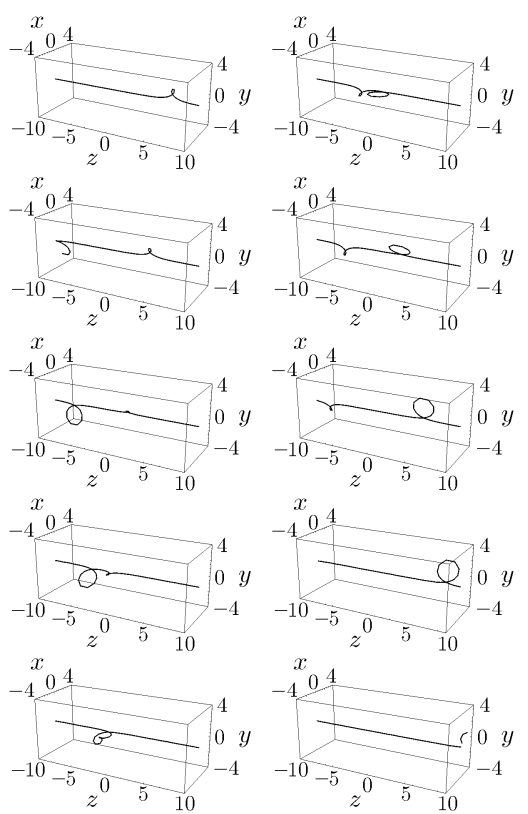

6

Рис. 3. Лобовое столкновение двух вращающихся солитонов, движущихся в противоположных направлениях с одинаковой скоростью $v$. Первый солитон движется направо, второй - налево. 
В случае взаимодействия между покоящимся и движущимся солитонами покоящийся солитон начинает двигаться, а движущийся становится покояшимся после взаимодействия, причем угол $\theta$ изменяется так, как показано на рис. 4 а для случая $v_{1}=0.12$, $\Omega_{1}=-0.1$. Если амплитуды солитонов сильно различаются, две петли взаимодействуют во время столкновений более сложным образом (см. рис. 4 для случая $v_{1}=0.36$, $\left.\Omega_{1}=-0.1\right)$.
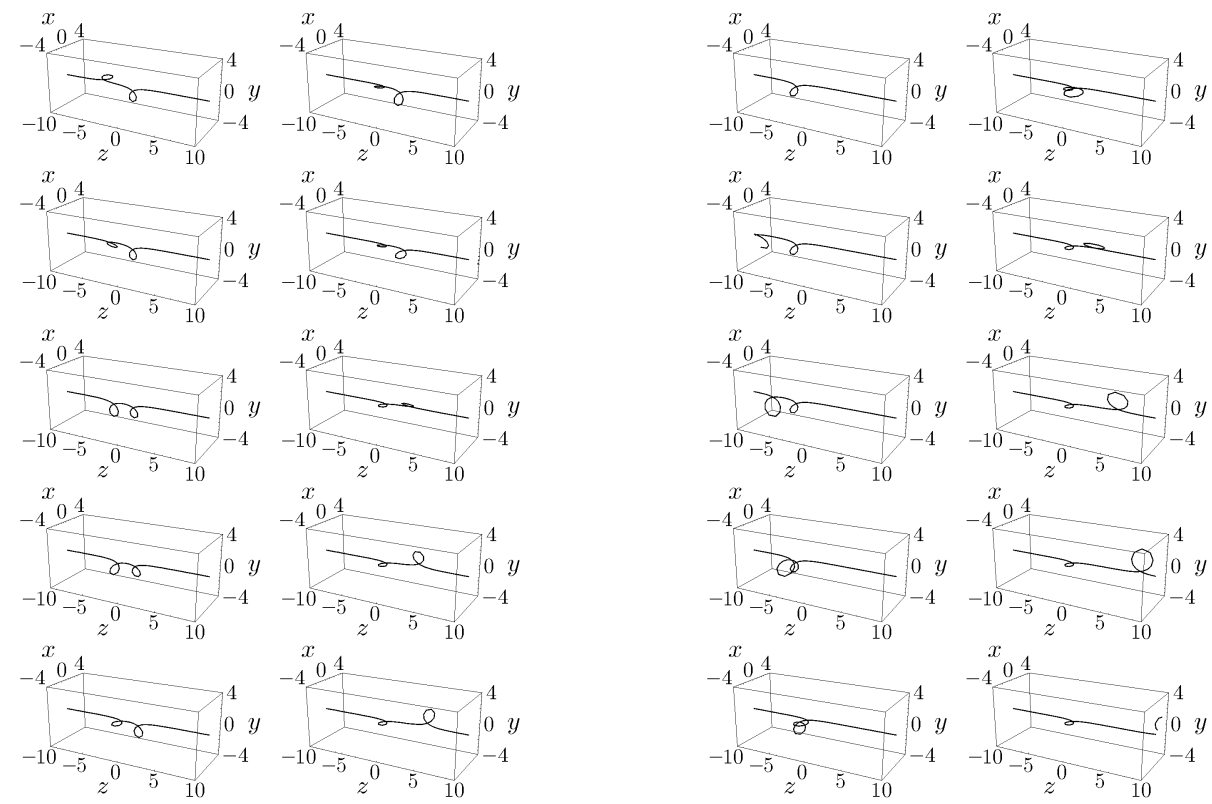

a
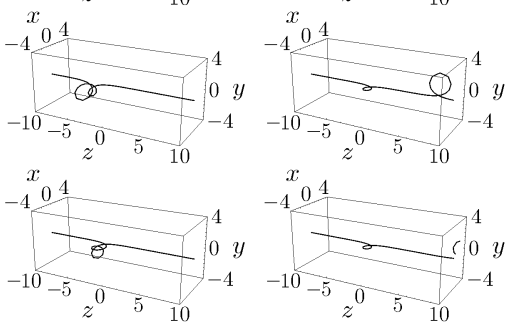

б

Рис. 4. Лобовое столкновение вращающегося солитона (с фазовой скоростью $v_{1}$ и угловой скоростью $\Omega_{1}$ ) с покоящимся. Первый солитон движется, второй солитон покоится.

\section{4. ЗАКЛЮЧИТЕЛЬНЫЕ ЗАМЕЧАНИЯ}

Придерживаясь той точки зрения, что связанные бездисперсионные уравнения представляют токоподводящую струну, движущуюся в трехмерном евклидовом пространстве и взаимодействующую с внешним магнитным полем, мы привели новый тип петлевых солитонов этих уравнений. Мы ожидаем, что петлевую деформированную струну удастся наблюдать экспериментально.

Мы получили врашаюшиеся одно- и двухсолитонные решения из модифицированных билинейных уравнений. Солитоны распространяются в трехмерном пространстве $\mathbb{R}^{3}$ с ненулевыми моментами импульса. Хотя рассмотренная система является $(1+1)$-мерной, солитоны имеют трехмерные характеристики. Были приведены несколько случаев взаимодействия между солитонами. Эффект врашения солитонов влечет за собой искажения петель во время взаимодействия. Подробному рассмотрению взаимодействия солитонов будет посвящена отдельная работа. 
В завершение мы хотели бы отметить, что билинейные уравнения (7) или уравнение (15) можно легко обобщить на случай с более чем тремя компонентами. Полезно вывести солитоные решения для обобшенных бездисперсионных систем, описанных в работе [7], которые являются многокомпонентным вариантом уравнений (2), по крайней мере с двухсолитонным решением.

Благодарности. Настояшая работа выполнена при частичной поддержке Grandin-Aid для научных исследований Министерства образования, культуры, спорта и технологии Японии. Х.Какухата благодарит Дойо-кай за ценные обсуждения.

\section{Список литературы}

[1] H. Kakuhata, K. Konno. J. Phys. Soc. Japan. 1996. V. 65. P. 340.

[2] H. Kakuhata, K. Konno. J. Phys. A. 1997. V. 34. P. L401; V. P. Kotlyarov. J. Phys. Soc. Japan. 1994. V. 63. P. 3535; H. Kakuhata, K. Konno. J. Phys. Soc. Japan. 1996. V. 65. P. 1. [3] K. Konno. Appl. Anal. 1995. V. 57. P. 209.

[4] H. Kakuhata, K. Konno. J. Phys. Soc. Japan. 1999. V. 68. P. 757.

[5] T. Alagesan, K. Porsezian. Chaos, Solitons, and Fractrals. 1996. V. 7. P. 1209; 1997. V. 8. P. 1645 .

[6] K. Konno, A. Jeffrey. The loop soliton. In: Advances in Nonlinear Waves (Res. Notes Math. Vol. 95). V. 1. Ed. L. Debnath. Boston: Pitman, 1984. P. 162.

[7] K. Konno, H. Kakuhata. J. Phys. Soc. Japan. 1996. V. 65. P. 713. 